\title{
Analytic continuation and fixed points of the Poincaré mapping for a polynomial Abel equation
}

Received March 31, 2006 and in revised form December 12, 2006

\begin{abstract}
We consider an Abel differential equation $y^{\prime}=p(x) y^{2}+q(x) y^{3}$ with $p(x), q(x)$ polynomials in $x$. For two given points $a$ and $b$ in $\mathbb{C}$, the "Poincaré mapping" of the above equation transforms the values of its solutions at $a$ into their values at $b$. In this paper we study global analytic properties of the Poincaré mapping, in particular, its analytic continuation, its singularities and its fixed points (which correspond to the "periodic solutions" such that $y(a)=y(b)$ ). On the one hand, we give a general description of singularities of the Poincaré mapping, and of its analytic continuation. On the other hand, we study in detail the structure of the Poincaré mapping for a local model near a simple fixed singularity, where an explicit solution can be written. Yet, the global analytic structure (in particular, the ramification) of the solutions and of the Poincaré mapping in this case is fairly complicated, and, in our view, highly instructive. For a given degree of the coefficients we produce examples with an infinite number of complex "periodic solutions" and analyze their mutual position and branching. Let us recall that Pugh's problem, closely related to the classical Hilbert's 16th problem, asks for the existence of a bound on the number of real isolated "periodic solutions". New findings reported here lead us to propose new insights on the Poincaré mapping. If the "complexity" of the path in the $x$-plane between $a$ and $b$ is $a$ priori bounded, the number of fixed points should be uniformly bounded. We think that, in some sense, this is close to the complex version of Khovanskiî's fewnomial theory.
\end{abstract}

\section{Introduction}

In this paper we investigate some global analytic properties of the "Poincaré mapping" $\phi$ for an Abel differential equation of the form

$$
y^{\prime}=p(x) y^{2}+q(x) y^{3} .
$$

For two given points $a$ and $b, \phi$ transforms the values $y(a)$ of the solutions $y(x)$ of this equation at $a$ into their values $y(b)$ at $b$. A more precise definition is given in Section 3 below.

The "compactification at infinity" $Y=1 / y$ transform the above equation to the form $d x / d t=Y, d y / d t=p(x) Y+q(x)$, which is a generalized Liénard equation. Essentially, we study the geometry of the complex foliation, produced by this equation.

J.-P. Francoise: Université P.-M. Curie, Paris 6, Laboratoire J.-L. Lions, UMR 7589 CNRS,

175 Rue de Chevaleret, 75013 Paris, France; e-mail: jpf@ math.jussieu.fr

N. Roytvarf, Y. Yomdin: Department of Mathematics, The Weizmann Institute of Science, Rehovot 76100, Israel; e-mail: nina.roytvarf@weizmann.ac.il, yosef.yomdin@weizmann.ac.il 
However, we follow the approach of the classical analytic theory of differential equations (see [37, 25, 29, 35]) and consider the solutions of the Abel equation and its Poincaré mapping as ramified analytic functions of one complex variable. Consequently, the main questions under investigation are fixed and movable singularities, analytic continuation and ramification of the functions involved. An especially important role in our analysis is played by the study of the continuation paths and their evolution.

Below we always restrict ourselves to the case of a polynomial Abel equation

$$
y^{\prime}=p(x) y^{2}+q(x) y^{3}, \quad y(0)=y_{0},
$$

with $p(x), q(x)$ polynomials in the complex variable $x$ of degrees $d_{1}, d_{2}$ respectively.

The following two problems for equation (1.1) have been intensively studied (see [2]-[5], [8]-[12]), [15]-[19], [28, 34, 41]:

a. For given $a, b$ is it possible to bound the number of real solutions $y(x)$ of (1.1), satisfying $y(a)=y(b)$, in terms of the degrees $d_{1}, d_{2}$ only?

b. Is it possible to give explicit conditions on $p$ and $q$ for the identity $y(a) \equiv y(b)$ to be satisfied for all the solutions of (1.1)?

These two problems are well known to be closely related to the classical Hilbert's 16th problem (second part; see, for example, [27]) and Poincaré's center-focus problem for polynomial vector fields on the plane.

Adopting the standard terminology in these problems, we shall call solutions of (1.1), satisfying $y(a)=y(b)$, closed or periodic, and the conditions on $p, q, a, b$ for $y(a) \equiv$ $y(b)$ the center conditions. Let us stress that in general these notions depend on a continuation path from a to $b$.

Abel equations were first investigated and studied by Abel himself as natural extensions of Riccati equations. Abel found several examples which are integrable ([1]). Then this list was enriched by Liouville. Classical references are [1, 33, 25, 29], and the modern references [35, 31, 14] have been instrumental for us.

The main motivation for our study comes from those classical examples of polynomial Abel equations which can be solved explicitly. Moreover, we mostly (but not always) restrict ourselves to the case where a first integral is a rational function.

However, the global analytic structure (in particular, the ramification) of the solutions and of the Poincaré mapping in these examples turns out to be fairly complicated, and, in our view, highly instructive. We study the singularities and the branching of the solutions and of the Poincaré mapping.

In particular, for a fixed degree of the coefficients, we produce, varying the parameters, examples with arbitrarily many and with an infinite number of "periodic solutions". We analyze the mutual position and branching of these periodic solutions. Following this analysis we suggest that the above "periodic solutions" (or "limit cycles") sit on different leaves of the Poincaré mapping. In other words, although the equality $y(0)=y(b)$ is satisfied for a large number of the initial values $y_{0}$, it is realized on more and more complicated continuation paths from a to $b$. 
This conjectural behavior of the complex "limit cycles" reminds the behavior of the solutions of "fewnomial" equations in the complex domain, as prescribed by the complex Khovanskiŭ fewnomials theory $([30])$.

Our attempt to better understand the global structure of the Poincare mapping for polynomial Abel equations is also motivated by the recent progress in the investigation in [8]-[12], [7, 13, 15, 38, 41] of the center-focus problem for (1.1). As mentioned above, this problem is to give explicit conditions on $p$ and $q$ for the Poincaré mapping on $a, b$ to be identical, i.e. for $y(a) \equiv y(b)$. In particular, in [8]-[12] the moment and composition conditions, providing a close approximation of the center conditions, have been introduced. On this base in [7] complete "local center conditions" have been obtained, and the "local Bautin ideal" has been computed for the Poincaré mapping $\phi$, while in [13] similar conditions "at infinity" have been found.

Via Bautin's approach [6], further developed in [22, 23, 42], the knowledge of the Bautin ideal of the Poincare mapping $\phi$ allows one to produce "semi-local" bounds on the fixed points of $\phi$. In other words, we get a fairly accurate control of the fixed points inside the disk of convergence of the Taylor series of $\phi$ at the origin. It was already mentioned that the problem of the global control of real fixed points of $\phi$ is very closely related to the Hilbert 16th problem of counting limit cycles of plane vector fields.

However, the methods of [22, 23, 42] are at present strictly limited to the disk of convergence of $\phi$. Any attempt to "globalize" the information produced by these methods will require a much better understanding of the global analytic nature of $\phi$, in particular, of its analytic continuation, its singularities and its ramification structure. In this paper we start an investigation in this direction.

The paper is organized as follows:

In Section 2 we restate some classical results of [37] which provide the description of singularities of the solutions of (1.1). Our formulations of these results are somewhat more "quantitative" than the classical ones, providing accurate estimates of the domains and parameters involved. We also provide some lemmas relating the position of the singularities of the solutions of (1.1) to the initial values of these solutions. We do not give the proofs of these results in the present paper, referring the reader to [21].

On this base, in Section 3, we give a precise definition of the Poincaré mapping $\phi$, and discuss the problem of the analytic continuation of $\phi$. In particular, we give a constructive procedure for this analytic continuation, based on the path deformation following the movable singularities of the solutions.

In Section 4 we describe typical singularities of the Poincaré mapping $\phi$. This completes our general description of the Poincaré mapping for the Abel equation.

In Section 5 we produce, via a linearization, a "local model" of the Abel equation near a simple fixed singularity. It turns out to be the equation

$$
y^{\prime}=y^{2}+c x y^{3} .
$$

We produce its explicit solutions, and formulate some problems concerning this equation, which are investigated in the rest of the paper.

In Section 6 we analyze in detail one specific case $c=1 / 4$ of equation (1.2). The value $c=1 / 4$ is a "discriminant" value of the parameter $c$, so the solution takes a special 
form, which in some aspects is easier to investigate than the case of a generic $c$. We study for $c=1 / 4$ the singularities and ramification of the solutions and of the Poincare mapping, describing, in particular, the fundamental domain and the monodromy action for the latter. Finally, we combine these results to produce an infinite number of periodic solutions and to analyze their geometry.

In Sections 7-9 we analyze in detail the case $c=c_{n}=\frac{1}{4}\left(1-\frac{1}{(2 n+1)^{2}}\right)$, where the first integral is a rational function. We follow the same pattern as in Section 7, ultimately producing $n$ periodic solutions on different leaves of the Poincaré mapping.

\section{The Abel equation}

Below we shall always assume that the functions $p(x)$ and $q(x)$ in the Abel equation (1.1) are polynomials in $x$ with complex coefficients. Most of the results below remain valid for much more general analytic functions, but our assumption simplifies the presentation.

Let $a \in \mathbb{C}$. Denote by $y\left(y_{a}, x\right)$ the solution of (1.1) satisfying $y\left(y_{a}, a\right)=y_{a}$. By the uniqueness and existence results for ordinary differential equations, the solution $y\left(y_{a}, x\right)$ exists in a certain neighborhood of $a$ and is there a regular complex analytic function of the complex argument $x$. However, an analytic continuation of $y\left(y_{a}, x\right)$ may lead to singularities.

The classical result of Painlevé [37] shows that the "movable" singularities of the solutions $y\left(y_{a}, x\right)$ must be "algebroid". Moreover, following the proof of Painlevé (see, for example [37, 25, 35]), one can easily show that at each movable singular point $x_{0}, y\left(y_{a}, x\right)$ behaves like $1 / \sqrt{x-x_{0}}$. In order to relate the singularities of $y$ to those of the Poincaré mapping $\phi$ we need more detailed information on the position of the singularities, on their dependence on the initial values, etc., than is usually given. So below we restate in the special case of (1.1) the classical results, providing some specific estimates where necessary.

Notice that $y \equiv 0$ is a solution of (1.1). It follows, in particular, that as $y_{a} \rightarrow 0$ all the singularities of $y\left(y_{a}, x\right)$ tend to infinity. Below we make this remark more precise.

Another remark is that, as we shall see below, the problematic points of the equation (1.1) are the zeroes of $q(x)$. We denote these zeroes by $x_{1}, \ldots, x_{m}$ and always distinguish between the "fixed" singularities of $y$ at $x_{1}, \ldots, x_{m}$ and the "movable" singularities of $y$, which may occur at points different from $x_{1}, \ldots, x_{m}$.

\subsection{Domain of regularity of solutions}

The following assumptions will be preserved along the rest of this section: $p(x)$ and $q(x)$ are polynomials of degree $m$ in $x$, with $\|p\|,\|q\| \leq K$. The norm of a polynomial is defined here as the sum of the absolute values of its coefficients. Let $a \in \mathbb{C}$. Denote, as above, by $y\left(y_{a}, x\right)$ the solution of (1.1) satisfying $y\left(y_{a}, a\right)=y_{a}$.

Lemma 2.1. Let $a \in \mathbb{C}$ and $y_{a} \in \mathbb{C}$ be given. Then the solution $y\left(y_{a}, x\right)$ exists in a disk $D_{\rho}(a)$ centered at a. Here $\rho=\rho\left(|a|,\left|y_{a}\right|\right)$ is a positive explicitly given function of its 
arguments, which for $\left|y_{a}\right|$ large satisfies

$$
\rho\left(|a|,\left|y_{a}\right|\right) \geq C_{1}\left(4 K|a|^{m}\left|y_{a}\right|^{2}\right)^{-1} .
$$

For $\left|y_{a}\right|$ small, $\rho$ satisfies

$$
\rho\left(|a|,\left|y_{a}\right|\right) \geq C_{2}\left(\frac{1}{2 K\left|y_{a}\right|}\right)^{1 /(m+1)} .
$$

In particular, $\rho$ tends to infinity as $\left|y_{a}\right|$ tends to zero.

The solution $y\left(y_{a}, x\right)$ is bounded in the disk $D_{\rho}(a)$ by $\hat{y}\left(\left|y_{a}\right|,|a|,|x-a|\right)$, with $\hat{y}$ an explicitly given function of its arguments satisfying

$$
\hat{y}\left(\left|y_{a}\right|,|a|, t\right) \leq C_{3}\left(\left|y_{a}\right|,|a|\right)(\rho-t)^{-1 / 2} .
$$

Proof. We do not give the proofs of the statements in this section, referring the reader to [21].

Corollary 2.1. $y\left(y_{a}, x\right)$ is regular in the disk $D_{R}$ of radius $R$ centered at the origin, with $R$ growing as $\left(2 K\left|y_{a}\right|\right)^{-m-1}$ as $\left|y_{a}\right|$ tends to zero.

\subsection{Singularities of $y\left(y_{a}, x\right)$}

In this subsection we restate the classical results on the structure of singularities of $y\left(y_{a}, x\right)$, stressing the explicit estimates of the size of the domains where the results are valid. The assumptions on $p$ and $q$ and the notations remain the same as in Subsection 2.1.

First we recall the following simple property of singular points of solutions $y(x)$ of (1.1):

Lemma 2.2. If $x_{0} \in \mathbb{C}$ is a singular point of a solution $y(x)$ of (1.1), then $y$ tends to infinity as $x$ tends to $x_{0}$.

Next we give an analytic description of the movable singular points of the solutions of (1.1). As already mentioned in the Introduction, the classical result of Painlevé [37, 25, 35] shows that "movable" singularities of the solutions $y\left(y_{a}, x\right)$ of (1.1) must be "algebroid". Moreover, following the proof of Painlevé (see, for example [37, 25, 35]), one can easily show that at each movable singular point $x_{0}, y\left(y_{a}, x\right)$ behaves like $1 / \sqrt{x-x_{0}}$. However, in order to relate the singularities of $y$ to those of the Poincare mapping $\phi$ we need more accurate estimates than are usually given, and in particular, we have to describe the behavior of the movable singular points of the solutions of (1.1) as functions of the initial value $y_{a}$. So we restate below the classical result of Painlevé (in the special case of the equation (1.1)), providing the required formulas and estimates.

To simplify the statements of the results below, let us introduce some notations. Assume that $x_{0} \in \mathbb{C}$ is different from the zeroes $x_{1}, \ldots, x_{m}$ of $q(x)$. Let $\eta\left(x_{0}\right)=$ $\left|q\left(x_{0}\right)\right|>0$. Put $R\left(x_{0}\right)=2\left(\left|x_{0}\right|+1\right)$ and define

$$
r\left(x_{0}\right)=\min \left(\frac{1}{4} R, \frac{\eta}{2 m(K+1) R^{m-1}}\right),
$$


where $K=\max \|p\|,\|q\|$. Finally, define

$$
M\left(x_{0}\right)=\frac{4 m(K+1) R^{m}}{\eta}, \quad \delta\left(x_{0}\right)=\frac{1}{M}, \quad c\left(x_{0}\right)=\frac{4}{M \eta}=\frac{1}{m(K+1) R^{m}} .
$$

Theorem 2.1. For any $x_{0} \in \mathbb{C}$ different from the zeroes $x_{1}, \ldots, x_{m}$ of $q(x)$, there is a unique solution $y(x)=y^{\left[x_{0}\right]}(x)$ of $(1.1)$ with a singularity at $x_{0}$. This solution has an algebraic ramification of order 2 at $x_{0}$. In a neighborhood of $x_{0}$, the solution is given by a Puiseux series

$$
y^{\left[x_{0}\right]}(x)=\frac{c\left(x_{0}\right)}{\left(x-x_{0}\right)^{1 / 2}} \cdot\left(1+\sum_{k=1}^{\infty} c_{k}\left(x_{0}\right)\left(x-x_{0}\right)^{k / 2}\right),
$$

converging for $\left|x-x_{0}\right| \leq r\left(x_{0}\right)$, with the coefficients $c\left(x_{0}\right), c_{k}\left(x_{0}\right)$ single-valued analytic functions in $\mathbb{C} \backslash\left\{x_{1}, \ldots, x_{m}\right\}$, satisfying there $\left|c_{k}\left(x_{0}\right)\right| \leq \delta\left(x_{0}\right)\left(r\left(x_{0}\right)\right)^{-k}$.

\subsection{Singularities of $y\left(y_{a}, x\right)$ as functions of $y_{a}$}

To relate the singularities of the Poincare mapping to those of the solutions of (1.1), it is important to see how the initial value of the solution $y$ at a certain regular point influences the position of the singularities of $y$. The description of the singularities of $y$, given above, allows one to get a rather accurate information in this respect.

Let us fix a certain point $c \in \mathbb{C}, c \neq x_{1}, \ldots, x_{m}$ (i.e. $q(c) \neq 0$ ).

Lemma 2.3. For any $y_{c}$ sufficiently large in absolute value, the solution $y\left(y_{c}, x\right)$ of $(1.1)$ satisfying $y\left(y_{c}, c\right)=y_{c}$ has a singularity $x_{0}=x_{0}\left(y_{c}\right)$ in a neighborhood of $c$. The position of this singularity, $x_{0}\left(y_{c}\right)$, is a regular function of $y_{c}$ for $\left|y_{c}\right|$ sufficiently large, and $d x_{0} / d y_{c} \neq 0$.

Corollary 2.2. Let the solution $y\left(y_{a}, x\right)$ of (1.1) satisfying $y\left(y_{a}, a\right)=y_{a}$ and continued to $x_{0} \neq x_{1}, \ldots, x_{m}$ along a path $\mathrm{s}$ in $\mathbb{C} \backslash\left\{x_{1}, \ldots, x_{m}\right\}$ have a singularity at $x_{0}$. Then $x_{0}$ is a regular function of the initial value $y_{a}$, and $d x_{0} / d y_{a} \neq 0$.

We can extend Lemma 2.3 above and describe the dependence of the position $x_{0}$ of the singular point of the solution on the value of this solution at the "original singular point" itself. Let us fix, as above, a certain point $c \in \mathbb{C}, c \neq x_{1}, \ldots, x_{m}$ (i.e. $q(c) \neq 0$ ).

Proposition 2.1. For $y_{c}$ near $\infty$, the position $x_{0}\left(y_{c}\right)$ of the singularity of the solution $y\left(y_{c}, x\right)$ of (1.1) satisfying $y\left(y_{c}, c\right)=y_{c}$ can be represented by a convergent Taylor series in $u=u(c)=1 / y(c)$,

$$
x_{0}-c=u^{2} \sum_{k=0}^{\infty} \alpha_{k} u^{k}, \quad \alpha_{0} \neq 0 .
$$

Conversely, the value $y\left(y_{c}, c\right)=y_{c}$ at $c$ of the solution $y$ of (1.1) having a singularity at $x_{0}$ can be represented by a convergent fractional Puiseux series

$$
u=u(c)=\sum_{k=1}^{\infty} \beta_{k}\left(x_{0}-c\right)^{k / 2}, \quad \beta_{1} \neq 0 .
$$


Below we shall use this proposition to describe the ramification of the Poincare mapping around its singularities.

\section{Analytic continuation of the Poincaré mapping}

In this section we give a precise definition of the Poincaré mapping $\phi$ of the Abel equation

$$
y^{\prime}=p(x) y^{2}+q(x) y^{3}
$$

and discuss some related problems. Then we give a "semi-constructive" description of the analytic continuation of $\phi$ along a given path.

Let $a, b \in \mathbb{C}, b \neq x_{1}, \ldots, x_{m}$, where $x_{1}, \ldots, x_{m}$ are, as above, all the zeroes of $q$. Notice that if a solution $y$ of (1.1) happens to have a singularity at one of the $x_{i}, i=1, \ldots, m$, then the analytic structure of this solution near $x_{i}$ may be much more complicated than that described in Section 2 above (see examples in Section 5 below).

Let $s$ be a path in $\mathbb{C}$ joining $a$ and $b$. We do not assume that $s$ avoids the points $x_{1}, \ldots, x_{m}$, unless explicitly stated. Let the initial value $y_{a}^{0} \in \mathbb{C}$ be given. Assume that the solution $y\left(y_{a}^{0}, x\right)$ of (1.1) satisfying $y\left(y_{a}^{0}, a\right)=y_{a}^{0}$ can be analytically continued along $s$ from a neighborhood of $a$. In particular, this continuation does not have singularities on $s$.

Definition 3.1. The (germ at $y_{a}^{0}$ of the) Poincaré mapping $\phi=\phi_{a, b, s, y_{a}^{0}}$ of the equation (1.1) along the path $s$ is defined as follows: it associates to each $y_{a}$ near $y_{a}^{0}$ the value $y_{b}$ at the point $b$ of the solution $y\left(y_{a}, x\right)$ of (1.1) satisfying $y\left(y_{a}, a\right)=y_{a}$ and continued to $b$ along $s$. Thus $\phi\left(y_{a}\right)=y\left(y_{a}, b\right)=y_{b}$.

Since $y \equiv 0$ is a solution of (1.1), the germ of the Poincare mapping at zero satisfies $\phi(0)=0$ along any path $s$ and for any endpoints $a, b$. Moreover, by Corollary 2.1, for any $R>0$ the solutions $y\left(y_{a}, x\right)$ are regular in the disk $D_{R}$, assuming that $\left|y_{a}\right|$ is sufficiently small. Hence, for any $a, b, s$, the germ at the origin of $\phi_{a, b, s}$ is defined and it does not depend on the path $s$.

However, for larger values of $y_{a}$, analytic continuation of $y\left(y_{a}, x\right)$ along different paths $s$ may lead to different values of $y_{b}$.

Now assume that a path $\sigma$ from $w_{0}=\sigma(0)$ to $w_{1}=\sigma(1)$ in the plane of the initial values $y_{a}$ is given, parametrized by $t \in[0,1]$. Assuming that none of the solutions $y\left(w_{t}, x\right), w_{t}=\sigma(t), t \in[0,1]$, has a singularity on the path $s$, the definition above works and defines the values (in fact, the germs) of $\phi\left(w_{t}\right), t \in[0,1]$, and, in particular, $\phi\left(w_{1}\right)$.

The problem appears if the singularities of the solutions $y\left(w_{t}, x\right)$ continued along $s$ approach and cross the path $s$. The idea of the following construction is that if we can deform the path $s$ (following the movement of $w_{t}$ along the curve $\sigma$ ) in such a way that it avoids the singularities of $y\left(w_{t}, x\right)$, we can still use this deformed path for analytic continuation of the solutions $y(w, x)$, and hence for analytic continuation of $\phi$.

Let $\sigma$ as above be given. Assume that there exists a family $s_{t}, t \in[0,1]$, of paths from $a$ to $b$ with the following properties:

1. $s_{t}$ is a continuous (in $t$ ) deformation of the original path $s, s_{0}=s$. 
2. For each $t \in[0,1]$, the solution $y\left(w_{t}, x\right)$ continued along $s_{t}$ is regular at each point of $s_{t}$.

Theorem 3.1. The germ of the Poincaré mapping along $s$ at the point $w_{0}, \phi_{a, b, s, w_{0}}$, admits analytic continuation along $\sigma$ from $w_{0}=\sigma(0)$ to $w_{1}=\sigma(1)$. The continued germ at $w_{1}$ is $\phi_{a, b, s_{1}, w_{1}}$ provided by the analytic continuation of the solutions starting near $w_{1}$ along $s_{1}$.

Proof. We shall show that for each $t \in[0,1]$ the value (germ) of $\phi\left(w_{t}\right)$, obtained by analytic continuation of $\phi$ along $\sigma$, is given by

$$
\phi\left(w_{t}\right)=y\left(w_{t}, b\right),
$$

with the solution $y\left(w_{t}, x\right)$ satisfying $y\left(w_{t}, a\right)=w_{t}$ being analytically continued from $a$ to $b$ along the path $s_{t}$. We can subdivide the process of analytic continuation of $\phi$ into a finite number of small successive steps. In each step we first move $w_{t}$ along $\sigma$, without deforming $s_{t}$ (provided that the singularities of $y\left(w_{t}, x\right)$ do not hit $s_{t}$ ). Then we deform $s_{t}$ without changing $w_{t}$. Clearly, the first part of each step gives an analytic continuation of $\phi$ along $\sigma$, while the second step does not change $\phi$ at all. Therefore the total procedure provides the required analytic continuation of $\phi$. This completes the proof of Theorem 3.1.

Theorem 3.1 reduces the problem of analytic continuation of the Poincaré mapping $\phi$ to the construction of a family of paths $s_{t}$ with the properties stated above.

One particular case is very easy: if the singularities of $y\left(w_{t}, x\right)$ do not approach the path $s$, it does not need to be deformed, and we can take $s_{t} \equiv s$.

In general, the construction of $s_{t}$ depends on the behavior of the singularities of the solutions $y\left(w_{t}, x\right)$. Below we describe it only in the simplest situation, where these singularities do not "escape to infinity" in finite time and cross the path $s$ transversally.

We assume, in particular, that the path $s$ avoids the "fixed" singularities $x_{1}, \ldots, x_{m}$ of (1.1). As usual, we assume that the solution $y\left(w_{0}, x\right)$ of (1.1) satisfying $y\left(w_{0}, a\right)=w_{0}$ can be analytically continued to $b$ along $s$ from a neighborhood of $a$. In particular, this continuation does not have singularities on $s$.

The following procedure (P) allows one to construct the required deformation $s_{t}$ of the initial path $s=s_{0}$. We consider the solutions $y\left(w_{t}, x\right)$ of $(1.1)$ for $w_{t}=\sigma(t)$ as $t$ moves from 0 to 1 . The singularities $x_{0}^{j}(t)$ of $y\left(w_{t}, x\right)$ move in this process along certain trajectories $\ell_{j}$. By Corollary 2.2 , as long as these singularities remain in $\mathbb{C} \backslash\left\{x_{1}, \ldots, x_{m}\right\}$, their positions depend in a regular way on the initial value $w_{t}$. Therefore, after a small deformation of $\sigma$ we can assume that the trajectories $\ell_{j}$ do not pass through $a$ and $b$, and that they cross $s$ transversally and at different times $t$.

Let $t_{0}$ be the earliest crossing time: for $t=t_{0}$ the trajectory $x_{0}^{i}(t)$ of the singularity $x_{0}^{i}$ crosses $s$ for the first time.

The main step of the procedure (P) consists in deforming $s$ in a small neighborhood of a crossing point of $s$ and $\ell_{i}$, to avoid the singularity $x_{0}^{i}(t)$ as it approaches and crosses $s$ along $\ell_{i}$ for $t$ near $t_{0}$, and then stretching $s$ along $\ell_{i}$, escaping the point $x_{0}^{i}(t)$ for $t>t_{0}$. This step is shown in Fig. 1. It defines the required deformation $s_{t}$ for $t$ from $t_{0}$ until the time of the next crossing. 


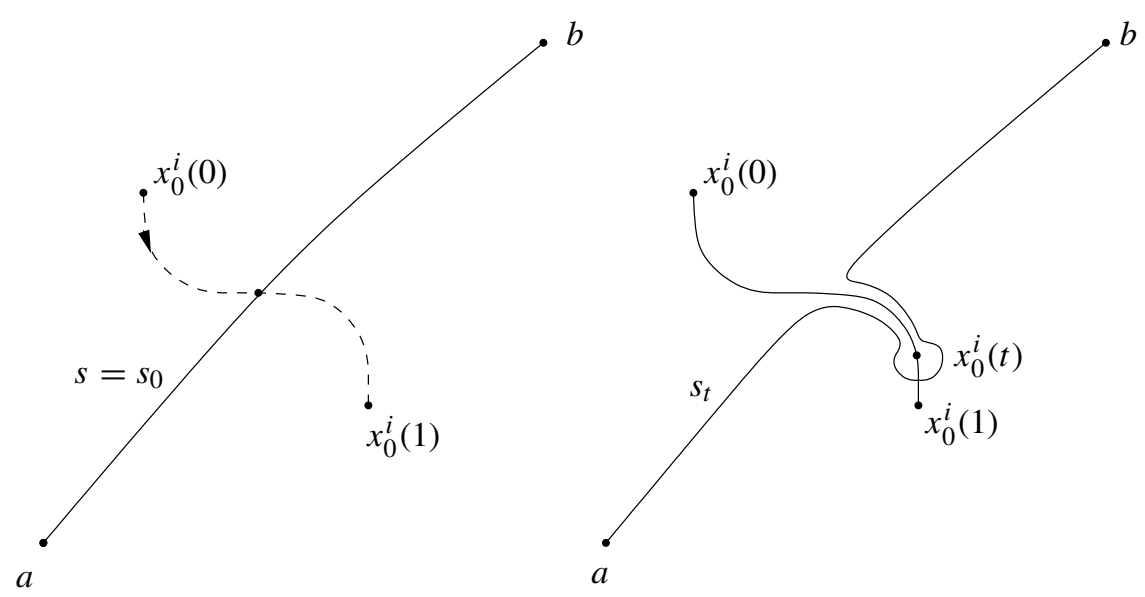

Fig. 1

When the next singularity $x_{0}^{j}(t)$ of $y\left(w_{t}, x\right)$ crosses the path $s_{t}$, we repeat the construction. Notice that from now on the crossing may happen at the "double lines" of $s_{t}$. In this case these double lines are deformed, as described above.

More precisely, this process can be described as follows: After the first crossing of $s$ by the singularity $x_{0}^{i}(t)$, the deformed path $s_{t}$ is obtained from $s$ by adding to $s$ the following loop $\delta_{t}^{i}: \delta_{t}^{i}$ starts at the crossing point $\omega_{i}$ of $s$ and $\ell_{i}$, follows $\ell_{i}$ till the point $x_{0}^{i}(t)$, encircles $x_{0}^{i}(t)$ in a counter-clockwise direction, and then returns to $\omega_{i}$ along $\ell_{i}$.

If the second crossing happens at a simple point of $s_{t}$, the new loop $\delta_{t}$ is added, exactly as above. If this crossing appears at a "double line" of $s_{t}$ (which belongs to the added loop $\delta_{t}^{i}$ ), the new loop $\delta_{t}$ is added to both branches of $s_{t}$ passing through the crossing point.

For the later crossings the process is repeated, with appropriate corrections, taking into account the "multiplicity" of the path $s_{t}$ at the crossing point.

One of the possible results of the deformation of $s$ via the procedure $(\mathrm{P})$ is shown in Fig. 2.

By the construction, the singular points $x_{0}^{j}(t)$ of the solutions $y\left(w_{t}, x\right)$ of (1.1) never cross the path $s_{t}$. On each crossing of the already constructed path with $x_{0}^{j}(t)$ we extend the deformation $s_{t}$ to a larger value of $t$, till the next crossing time.

Let us assume, in addition to the assumptions made above, that only a finite number of singularities are encountered in this process, and that the trajectory $\ell_{i}$ of none of these singularities $x_{0}^{i}(t)$ escapes to infinity. Then the following result is true:

Theorem 3.2. Under the above assumptions the procedure $(P)$ can be extended to the entire interval $[0,1]$, and it provides a deformation $s_{t}$ of the path $s$ as required in Theorem 3.1 .

If we know explicitly the trajectories $\ell_{j}(t)$ of the singular points $x_{0}^{j}(t)$, we can provide an explicit description of the final path $s$ as an element of the appropriate fundamental 


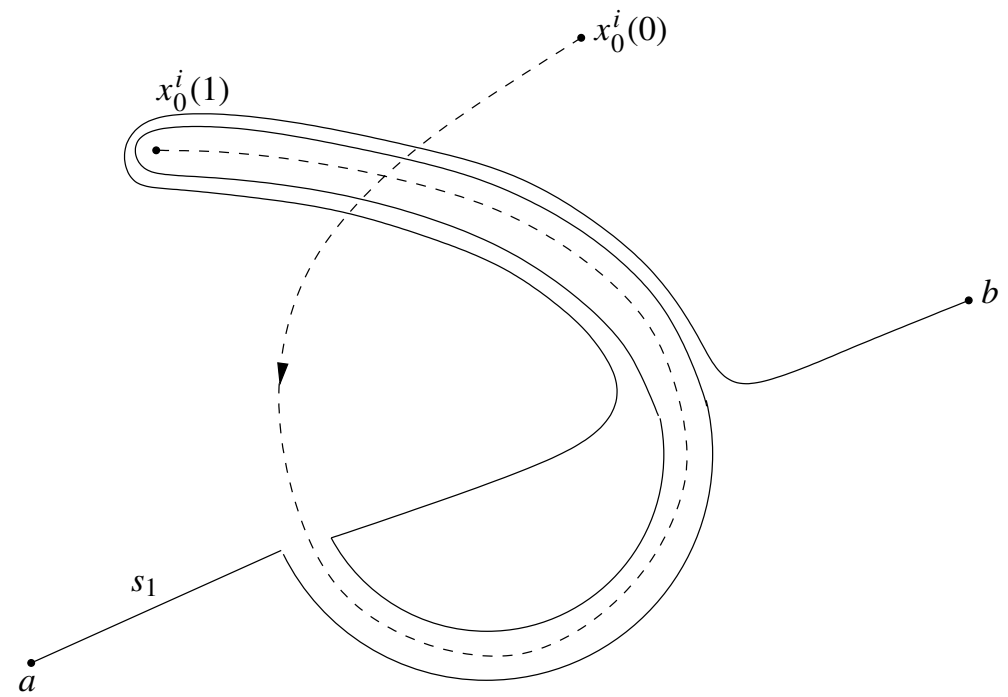

Fig. 2

group. Then an application of Theorem 3.1 provides an explicit analytic continuation of the Poincaré mapping $\Phi$ along $\sigma$. Below we illustrate this construction by some examples.

\section{Singularities of the Poincaré mapping}

Let $s$ be a path in $\mathbb{C}$ joining two points $a$ and $b$, and let the initial value $y_{a}^{0} \in \mathbb{C}$ be given. Assume that the solution $y\left(y_{a}^{0}, x\right)$ of the equation (1.1) satisfying $y\left(y_{a}^{0}, a\right)=y_{a}^{0}$ can be analytically continued along $s$ from a neighborhood of $a$ to each point of $s$ except, possibly, the endpoint $b$. In particular, this continuation does not have singularities at the interior points of $s$. If $b$ is also a regular point of this solution, then the germ at $y_{a}^{0}$ of the Poincaré mapping $\phi=\phi_{a, b, s, y_{a}^{0}}$ along the path $s$ is defined and regular.

Consider now the case when the analytic continuation along $s$ of the solution $y\left(y_{a}^{0}, x\right)$ has a singularity at $b$. From now on, we assume that $b$ is different from the fixed singularities $x_{1}, \ldots, x_{m}$.

Proposition 4.1. Under the above assumptions there is a germ of a real curve $\gamma \subset \mathbb{C}$ at $y_{a}^{0}$ such that for $y_{a} \notin \gamma$ the analytic continuation along $s$ of the solution $y\left(y_{a}, x\right)$ is regular at each point of $s$ including the endpoint $b$.

Proof. We are in the situation of Corollary 2.2 above. By this corollary, the position $x_{0}\left(y_{a}\right)$ of the singularity of the solution $y\left(y_{a}, x\right)$ near $b$ is a regular function of $y_{a}$ near $y_{a}^{0}$. So the curve $\gamma$ is formed by exactly those $y_{a}$ for which this singularity $x_{0}\left(y_{a}\right)$ belongs to $s$. By the description of the singularities of the solutions of (1.1) given in Section 2, $x_{0}\left(y_{a}\right)$ is the only singular point of the local branch of the solution $y\left(y_{a}, x\right)$ near $b$. On 
the other hand, by the assumptions, there are no singularities of the solution $y\left(y_{a}, x\right)$ on $s$ not in a neighborhood of $b$. Therefore, for $y_{a} \notin \gamma$ the analytic continuation along $s$ of the solution $y\left(y_{a}, x\right)$ is regular at each point of $s$, including the endpoint $b$. This completes the proof of the proposition.

Now we are ready to describe the generic singular points of the Poincaré mapping.

Theorem 4.1. Let $s$ be a path in $\mathbb{C}$ joining two points $a$ and $b \neq x_{1}, \ldots, x_{m}$, and let the initial value $y_{a}^{0} \in \mathbb{C}$ be given. Assume that the solution $y\left(y_{a}^{0}, x\right)$ of $(1.1)$ satisfying $y\left(y_{a}^{0}, a\right)=y_{a}^{0}$ can be analytically continued along s from a neighborhood of a to each point of s except the endpoint $b$, where this solution has a singularity. Then for each $y_{a}$ in a neighborhood of $y_{a}^{0}$ such that $y_{a} \notin \gamma$, where the curve $\gamma$ has been defined in Proposition 4.1, the germ at $y_{a}$ of the Poincaré mapping $\phi=\phi_{a, b, s, y_{a}}$ along the path $s$ is defined and regular. In a punctured neighborhood $U_{0}$ of $y_{a}^{0}$ these germs can be analytically continued across $\gamma$ to form a double-valued regular function $\phi$ in $U_{0}$ which admits a representation by a Puiseux series

$$
y(b)=\frac{1}{\sqrt{y_{a}-y_{a}^{0}}} \sum_{k=0}^{\infty} v_{k}\left(y_{a}-y_{a}^{0}\right)^{k / 2}, \quad \nu_{0} \neq 0,
$$

convergent in $U_{0}$.

Proof. The fact that for each $y_{a}$ in a neighborhood of $y_{a}^{0}$ such that $y_{a} \notin \gamma$, the germ at $y_{a}$ of $\phi$ is defined and regular, follows directly from Proposition 4.1. The possibility of analytic continuation of $\phi$ across $\gamma$ follows from the results of Section 3. The local form of $\phi$ near $y_{a}^{0}$ can be obtained in two ways.

The first one uses Corollary 2.2 and Proposition 2.1. By Corollary 2.2, the position $x_{0}\left(y_{a}\right)$ of the singularity (near $\left.b\right)$ of the solution $y\left(y_{a}, x\right)$ analytically continued along $s$ is a regular function of $y_{a}$. By Proposition 2.1, $u_{b}=1 / y_{b}$, where $y_{b}=y\left(y_{a}, b\right)$, as a function of $x_{0}$, is given by

$$
u=u_{b}=\sum_{k=1}^{\infty} \beta_{k}\left(x_{0}-b\right)^{k / 2}, \quad \beta_{1} \neq 0 .
$$

Substituting into this expression a regular function $x_{0}\left(y_{a}\right), x_{0}\left(y_{a}^{0}\right)=b$, we get

$$
u=u_{b}=\sum_{k=1}^{\infty} c_{k}\left(y_{0}-y_{a}^{0}\right)^{k / 2}, \quad c_{1} \neq 0 .
$$

Finally, expressing $y_{b}=1 / u_{b}$ through $u_{b}$ via (4.3), we get the required formula (4.1).

The second way to describe the ramification of the Poincare mapping $\phi$ at its singular point is via the results of Section 3. Let us fix a point $w$ in the complex plane of the initial values $y_{a}$, close enough to $y_{a}^{0}$. Let $\sigma$ be a path going from $w$ to a small neighborhood of $y_{a}^{0}$, then going around $y_{a}^{0}$ in a counter-clockwise direction, and then returning to the initial point $w$. Let $x_{0}(w)$ be the singularity (near $b$ ) of the solution $y(w, x)$ analytically continued along $s$. Since by Corollary 2.2, $x_{0}(w)$ is a regular function of $w$ (and since 


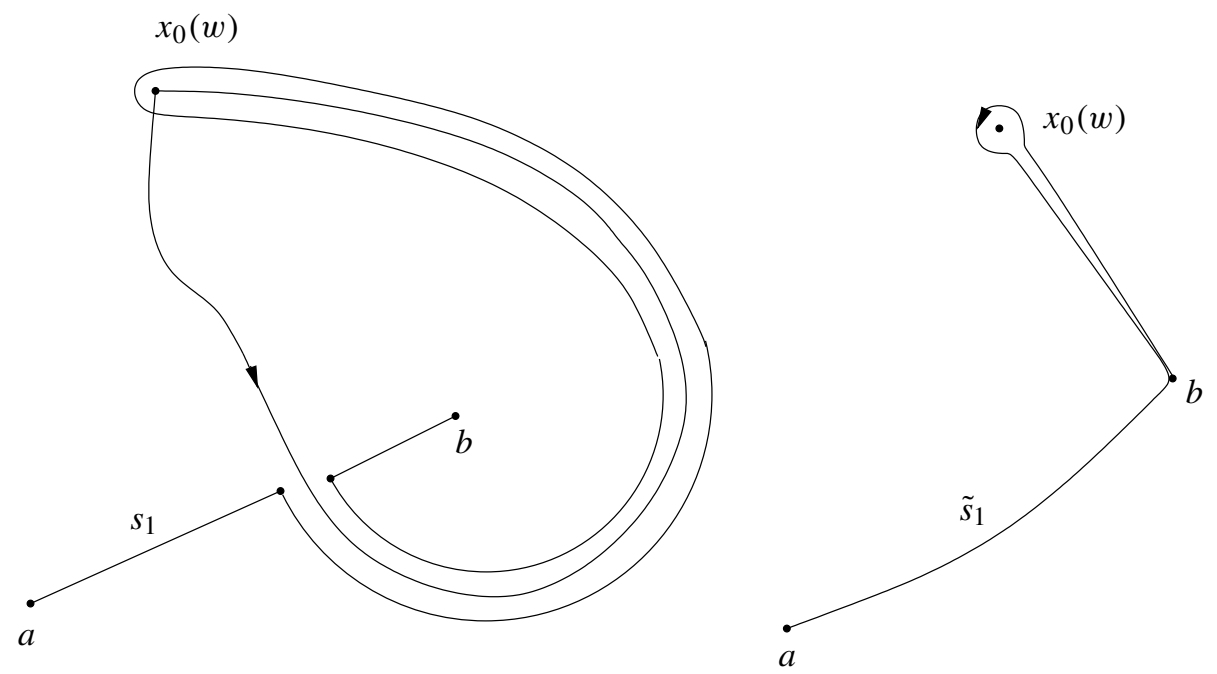

Fig. 3

the point $w$ was chosen close enough to $y_{a}^{0}$ ), as a running point $\tilde{w}$ moves along $\sigma$, the singularity $x_{0}(\tilde{w})$ of the solution $y(\tilde{w}, x)$ analytically continued along $s$ describes the path shown in Figure 3 above. In this simple situation all the assumptions of Theorem 3.2 are satisfied, and analytic continuation of the Poincaré mapping along $\sigma$ is provided by the deformed path $s_{1}$ shown in Figure 3 . In turn, the path $s_{1}$ is homotopic, in the complement of the singularities of $y(w, x)$, to the path $\tilde{s}_{1}$, also shown in Figure 3 . This path $\tilde{s}$ is obtained from $s$ by adding a loop going around the singularity $x_{0}(w)$ of the solution $y(w, x)$. But we know by the results of Section 2 that $y(w, x)$ has a ramification of order 2 at $x_{0}(w)$. Hence, as we follow $\sigma$ once, we return to another branch of $\phi$, but after the second tour we return to the initial branch of $\phi$, and therefore $\phi$ has a ramification of order two at $y_{a}^{0}$. This completes the proof of Theorem 4.1.

Remark. Let us describe once more the role of the curve $\gamma$ defined in Proposition 4.1 and appearing in Theorem 4.1 above. This curve $\gamma$ is formed by exactly those initial values $y_{a}$ for which the singularity $x_{0}\left(y_{a}\right)$ of the solution $y\left(x, y_{a}\right)$ analytically continued along the path $s$ belongs to $s$. Formally, the solutions starting at $y_{a} \in \gamma$ cannot be continued along $s$.

In the real case this presents a serious difficulty: all the real solutions, starting in a certain interval of values $y_{a}$, blow up inside the interval $[a, b]$, and there is no natural way to determine their value at $b$.

In the complex domain the curve $\gamma$ does not have any intrinsic meaning, since the continuation path $s$ can be deformed. The curve $\gamma$ just provides a natural "cut" of the neighborhood of the singularity $y_{a}^{0}$ of the Poincare mapping $\phi$. The analytic continuation of $\phi$ across $\gamma$ is described by Theorem 4.1. See the example of Section 8.1 below where the curve $\gamma$ is used as a global cut. 
Let us stress that the local branch of the Poincaré mapping $\phi$ at $y_{a}^{0}$ is regular along $\gamma$, and $y_{a}^{0}$ is its only singularity. In particular, the Puiseux expansion given by (4.1) is valid only at $y_{a}^{0}$.

\section{A local model: an example of an integrable Abel equation}

In this section we investigate the local behavior of the solutions near a generic fixed singularity. That is, we assume that the polynomial $q(x)$ has a simple zero.

\subsection{Linearization at infinity}

We consider the foliation defined by the Abel equation near $y=\infty$, or $1 / y=0$. We change accordingly the coordinate $y$ to $1 / y$, which yields

$$
\begin{aligned}
-\frac{1}{y^{2}} \frac{d y}{d x} & =q(x) \frac{1}{x^{3}}+p(x) \frac{1}{x^{2}}, \\
\frac{d y}{d x} & =\frac{q(x)+p(x) y}{-y} .
\end{aligned}
$$

This foliation is also defined by the vector fields

$$
\frac{d x}{d t}=-y, \quad \frac{d y}{d t}=q(x)+p(x) y,
$$

which is usually called the generalized Liénard equation. We can assume that $q$ has a simple zero at $x=0$ and that $p(0) \neq 0$. A new scaling of the variables $(x, y)$ and of the time $t$ displays the linear part of the vector field at $(0,0)$ as

$$
\frac{d x}{d t}=-y, \quad \frac{d y}{d t}=c x+y .
$$

Hence, the generalized Liénard vector field can be linearized near $(0,0)$. Back to the initial coordinates, this yields the local model

$$
\frac{d y}{d x}=c x y^{3}+y^{2} .
$$

\subsection{Solving the model equation}

According to the result of the previous section, we consider the equation

$$
\frac{d y}{d x}=c x y^{3}+y^{2}
$$


The change of unknown function $y=v / x$ yields

$$
-\frac{v}{x^{2}}+\frac{v^{\prime}}{x}=\frac{c v^{3}}{x^{2}}+\frac{v^{2}}{x^{2}}
$$

and thus

$$
v^{\prime}=\frac{1}{x}\left[c v^{3}+v^{2}+v\right],
$$

which obviously separates. Write

$$
\frac{1}{c v^{3}+v^{2}+v}=\frac{\alpha}{v}+\frac{\beta}{v-v_{1}}+\frac{\gamma}{v-v_{2}},
$$

with

$$
v_{1}=\frac{-1+\sqrt{1-4 c}}{2 c}, \quad v_{2}=\frac{-1-\sqrt{1-4 c}}{2 c},
$$

and

$$
\alpha=1, \quad \beta=\frac{1}{c v_{1}\left(v_{1}-v_{2}\right)}, \quad \gamma=\frac{1}{c v_{2}\left(v_{2}-v_{1}\right)} .
$$

Integrating equation (5.2) we get, for each solution $v(x)$,

$$
v\left(v-v_{1}\right)^{\beta}\left(v-v_{2}\right)^{\gamma}=K \cdot x
$$

for a certain constant $K$. Equivalently,

$$
y\left(x y-v_{1}\right)^{\beta}\left(x y-v_{2}\right)^{\gamma}=K,
$$

or

$$
y\left(1-\frac{x y}{v_{1}}\right)^{\beta}\left(1-\frac{x y}{v_{2}}\right)^{\gamma}=\frac{K}{v_{1}^{\beta} v_{2}^{\gamma}}=K^{\prime} .
$$

Notice that the only "fixed singularity" of the equation (5.1) is $x=0$. To start, let us take this point $x=0$ as the initial point $a$. Now, the constant $K^{\prime}$ in (5.3) is evaluated by setting $x=0$ and $y=y_{0}$, which yields $K^{\prime}=y_{0}$.

Therefore, the solution $y\left(y_{0}, x\right)$ of $(5.1)$ satisfying $y\left(y_{0}, 0\right)=y_{0}$ is given by

$$
y(x)\left(1-\frac{x y(x)}{v_{1}}\right)^{\beta}\left(1-\frac{x y(x)}{v_{2}}\right)^{\gamma}=y_{0} .
$$

Substituting into (5.4) the point $x=b$, we get the relation between $y_{b}=y(b)$ and $y_{0}$ in the form

$$
y_{0}=y_{b}\left(1-\frac{b y_{b}}{v_{1}}\right)^{\beta}\left(1-\frac{b y_{b}}{v_{2}}\right)^{\gamma} .
$$

In a certain sense, (5.5) provides an explicit expression for the Poincaré mapping $y_{0}=$ $\phi\left(y_{b}\right)$ of (5.1). 


\subsection{Main questions}

Equations (5.4) and (5.5) give a complete solution of the model equation (5.1). However, since these equations produce multivalued solutions, their interpretation is far from being straightforward.

In this paper we discuss (and in some cases answer) the following questions:

1. Ramification structure of the solution $y\left(y_{0}, x\right)$ of (5.1) given by equation (5.4).

2. Ramification structure of the Poincaré mapping $\phi$ of (5.1), given by equation (5.5). It turns out that the correct interpretation of (5.5) cannot be done without a careful analysis of the ramifications of the solution $y\left(y_{0}, x\right)$ of (5.1).

3. Finally, we are interested in the "limit cycles" of the equation (5.1), i.e. in its solutions $y(x)$ satisfying $y(0)=y(b)$. This relation together with (5.5) gives the following equation for the limit cycles:

$$
\left(1-\frac{b y_{0}}{v_{1}}\right)^{\beta}\left(1-\frac{b y_{0}}{v_{2}}\right)^{\gamma}=1 .
$$

The accurate immediate interpretation of (5.6) is that the algebraic curve $Y=Y_{y_{0}}$ defined by (5.4) passes through the points $\left(0, y_{0}\right)$ and $\left(b, y_{0}\right)$.

Certainly, this curve $Y$, parametrized as $y=y(x)$, satisfies the differential equation (5.1). But a priori we do not even know whether $Y$ is connected. So (5.6) by itself does not exclude the possibility that the points $\left(0, y_{0}\right)$ and $\left(b, y_{0}\right)$ belong to different leaves of the solutions of the differential equation (5.1). So we have to clarify the geometric interpretation of the "limit cycles", specifying the continuation paths from $a$ to $b$ for the solutions $y(x)$ satisfying $y(0)=y(b)$.

Below we choose some specific values for the free parameter $c$ in the model equation (5.1). In particular, in all the cases considered we show that in fact for $y_{0} \neq 0$ the curve $Y=Y_{y_{0}}$ is connected. This allows us to give the following interpretation to the equation (5.6): for each $y_{0}$ satisfying (5.6) there exists a path $s$ from 0 to $b$ such that the solution $y\left(y_{0}, x\right)$ can be analytically continued along $s$, and this continuation satisfies $y\left(y_{0}, b\right)=y_{0}$.

On this base we produce examples of (5.1) with an infinite (or finite, but arbitrarily large) number of complex "limit cycles".

In these examples we see that the equation (5.1) may have as many complex limit cycles as we wish, when the parameter $c$ varies, although the degree of the coefficients of this equation remains bounded. This phenomenon resembles (in a much simpler setting) the counterexample due to Yu. Il'yashenko to the Petrovski1-Landis claim ([26]). It should also be compared with the examples of differentiable Abel equations discussed by A. Lins Neto ([32]). Note that Khovanskii fewnomials theory, or rather, "additive complexity" arguments (see [30, 40]) imply that the number of real roots of equation (5.7) remains bounded independently of $n$. So that this example does not provide a counterexample to the real Hilbert-Pugh problem. 
Moreover, in all the cases considered we show that the above limit cycles "sit on different leaves" of the Poincare mapping. In other words, although the equality $y(0)=$ $y(b)$ is satisfied for a large number of initial values $y_{0}$, it is realized on more and more complicated continuation paths from a to $b$.

This is similar to the behavior of solutions of "fewnomial" equations in the complex domain, as prescribed by the complex Khovanskil fewnomials theory.

Accordingly, we may ask to what extent this property remains valid for the Poincaré mapping of a general polynomial Abel equation (1.1).

Our basic example (5.1) can be used to generate a family of similar examples by the change of independent variable, or "polynomial compositions". Compositions appear quite naturally in this area (see both the classics (Abel, Liouville,...) and more recent contributions [8]-[12]). Consider the Abel equations of the form

$$
y^{\prime}=c P(x) p(x) y^{3}+p(x) y^{3},
$$

where $p(x)$ is an arbitrary polynomial, and $P(x)$ is the anti-derivative of $p(x)$ which vanishes at $x=0$. The change of variables $w=P(x)$ brings (5.7) to the form

$$
\frac{d y}{d w}=c w y^{3}+y^{2}
$$

Applying the above analysis of this last equation, we see that the solution $y(x)$ to the equation (5.7) satisfying $y(0)=y_{0}$ solves the implicit algebraic equation

$$
y\left(1-\frac{P(x) y}{v_{1}}\right)^{\beta}\left(1-\frac{P(x) y}{v_{2}}\right)^{\gamma}=y_{0} .
$$

Hence also the limit cycles of (5.7) can be investigated in a similar way. Notice, however, that a special composition structure of the solutions of (5.7), namely, that each of its solutions $y$ can be represented as $y(x)=\tilde{y}(P(x))$ for $\tilde{y}(w)$ solving (5.8), implies the following: for any $a, b$ with $P(a)=P(b)$ we have $y(a) \equiv y(b)$.

\section{The case $c=1 / 4$}

We consider the case $c=1 / 4$ and the equation

$$
\frac{d y}{d x}=\frac{1}{4} x y^{3}+y^{2}
$$

With $y=v / x$, this equation yields

$$
\frac{d v}{d x}=\frac{1}{4 x}\left(v^{3}+4 v^{2}+4 v\right)
$$

which separates and gives the solution $y(x)$ corresponding to the initial data $y_{0}$ as the solution to

$$
\frac{y}{x y+2} e^{2 /(x y+2)}=\frac{e}{2} y_{0} .
$$


Periodic orbits correspond to solutions with $y(1)=y(0)=y$ of

$$
\frac{2}{y+2} e^{2 /(y+2)}=e .
$$

If we change variable by setting $y=\frac{2 \xi}{1-\xi}$, this yields

$$
1-\xi=e^{\xi}
$$

We write $\xi=x+i y$, and derive the two equations

$$
1-x=e^{x} \cos y, \quad-y=e^{x} \sin y .
$$

Note that if $(x, y)$ is a solution, then $(x,-y)$ is also a solution. Thus we can assume $y>0$. The second equation implies $\sin y<0$ and we restrict ourselves now to $y \in$ ]$(2 n+1) \pi,(2 n+2) \pi\left[\right.$. Now we plug $x=-\log \left(-\frac{\sin y}{y}\right)$ into the first equation. This yields

$$
F(y)=1+\log \left(-\frac{\sin y}{y}\right)+\frac{y}{\tan y}=0 .
$$

Then we note that $F(y) \rightarrow+\infty$ as $y \rightarrow(2 n+1) \pi$, and $F(y) \rightarrow-\infty$ as $y \rightarrow(2 n+2) \pi$. There is thus at least one solution (and in fact a single one) in the above interval. The Abel equation has thus infinitely many limit cycles:

Theorem 6.1. For $c=1 / 4$ equation (5.1) has infinitely many different "limit cycles", i.e. local solutions $y^{j}(x)$ at the origin, $j=1, \ldots, n$, and paths $s^{j}$ from 0 to 1 , such that each $y^{j}(x)$ after analytic continuation along $s^{j}$ satisfies $y(0)=y(b)$.

Let us give some initial considerations supporting the assumption that all these "limit cycles" are situated on different leaves of the Poincaré mapping for (5.1). They concern the geometry of the Riemann surface defined by the equation

$$
\frac{y}{x y+2} e^{2 /(x y+2)}=\frac{e}{2} y_{0}
$$

We introduce the coordinate $Y$ defined by

$$
x y=2(1 / Y-1),
$$

and fix $y_{0}=1 / e$, to simplify. The equation of the Riemann surface becomes

$$
(1-Y) e^{Y}=x
$$

The different sheets of the Riemann surface, seen as a cover of the $x$-plane, can be explicitly parametrized. Write $x=\alpha+i \beta$ and $Y=u+i v$. Then

$$
u=-\log \left(-\frac{v}{\alpha \sin v+\beta \cos v}\right),
$$


and the sheets are parametrized by the different solutions $v$ to the equation

$$
1+\log \left(-\frac{v}{\alpha \sin v+\beta \cos v}\right)+v \frac{\alpha \cos v-\beta \sin v}{\alpha \sin v+\beta \cos v}=0 .
$$

Comparing these expressions with those defining the limit cycles above, we see that the "fundamental domains" are essentially the same. The accurate analysis of the ramification of the Poincaré mapping requires the use of the Lambert $W$-functions, defined by $W(x) e^{W(x)}=x$. (See, for example, [16].) We plan to present this analysis separately.

7. The case $c=c_{n}=\frac{1}{4}\left(1-\frac{1}{(2 n+1)^{2}}\right)$

To study in detail the other possible patterns of ramification of solutions of the Abel equation (5.1), $\frac{d y}{d x}=c x y^{3}+y^{2}$, we choose the parameter $c$ in this equation in the following way. We would like $c$ to tend to $1 / 4$, which is a "discriminant point" for the denominator $c v^{3}+v^{2}+v$ appearing after separation of variables in (5.1). On the other hand, we want the first integral to be algebraic. So let us write

$$
1-4 c=\delta^{2}, \quad c=\frac{1}{4}-\frac{\delta^{2}}{4},
$$

where $\delta$ is small. In this case we obtain

$$
v_{1}=-\frac{2}{1+\delta} \approx-2+2 \delta, \quad v_{2}=-\frac{2}{1-\delta} \approx-2-2 \delta .
$$

For $\beta$ and $\gamma$ we get, respectively,

$$
\beta=-\frac{1+\delta}{2 \delta}, \quad \gamma=\frac{1-\delta}{2 \delta} .
$$

Notice that $\beta+\gamma=-1$. Thus, taking $\delta=-1 /(2 n+1)$ for $n$ an integer, we obtain $v_{1}=$ $-2-1 / n, v_{2}=-2+1 /(n+1), \beta=n, \gamma=-n-1$ and $c=c_{n}=\frac{1}{4}\left(1-1 /(2 n+1)^{2}\right)$. Let us fix this choice of $c$.

Remark. One can investigate the situation for another choice of the parameter $c$. If we pick the number $n$ above (not an integer anymore) in such a way that $n /(n+1)=k$ or $n=-k /(k-1)$, and let $k$ be a large integer, this corresponds to $n \approx-1$ or $c \approx 0$. We also have $\beta=n=-k /(k-1)$, and $\gamma=-n-1=1 /(k-1)$. The equation (5.4) takes the form

$$
y^{1-k}\left(1-\frac{x y}{v_{1}}\right)^{k}=y_{0}^{1-k}\left(1-\frac{x y}{v_{2}}\right) .
$$

while (5.6) takes the form

$$
\left(1-\frac{b y_{0}}{v_{1}}\right)^{k}=1-\frac{b y_{0}}{v_{2}} .
$$

The investigation of this case is important since as $c$ tends to zero, equation (5.1) tends to the integrable equation $y^{\prime}=y^{2}$, and the results can be compared with those obtained 
in the study of the "first variation" of the Abel equation $y^{\prime}=\epsilon x y^{3}+y^{2}$ at $\epsilon=0$ (cf. [9] [13]).

Let us return to the case $c=c_{n}$. The first integral (5.4) now takes the form

$$
y_{0}=\frac{y\left(1-x y / v_{1}\right)^{n}}{\left(1-x y / v_{2}\right)^{n+1}}=H(x, y) .
$$

In the rest of this section we investigate in some detail the solutions of the algebraic equation (7.3).

First of all, we notice that for $y_{0}=0$ we get two separate leaves: the straight line $Y_{0}^{0}=\{y \equiv 0\}$ and the hyperbola $Y_{0}^{1}=\left\{y=v_{1} / x\right\}$. Concerning the zero curves of $H(x, y)$, the first has multiplicity 1 , while the second has multiplicity $n$.

Another remark is that the pole locus of $H(x, y)$ is the hyperbola $Y_{\infty}=\left\{y=v_{2} / x\right\}$, which has multiplicity $n+1$. As we can expect, both the hyperbolas above are solutions of the differential equation (5.1). This can be checked by direct substitution.

\subsection{Critical points of $H(x, y)$}

Let us find the critical points of the function $H(x, y)$.

Lemma 7.1. All the critical points of the function $H(x, y)$ (in fact, all the points with $\left.H_{y}(x, y)=0\right)$ are situated on the hyperbola $Y_{0}^{1}=\left\{y=v_{1} / x\right\}$.

Proof. After differentiating $H$ with respect to $x$ and $y$, cancelling the common degrees of $1-x y / v_{2}$, equating the numerator to zero, and some computations, using, in particular, the identities

$$
\frac{n}{v_{2}}-\frac{n+1}{v_{1}}=0, \quad \frac{n}{v_{1}}-\frac{n+1}{v_{2}}=1,
$$

we obtain the following system of equations:

$$
\begin{aligned}
& \left(1-\frac{x y}{v_{2}}\right)^{n} H_{y}=\left(1-\frac{x y}{v_{1}}\right)^{n-1}=0 \\
& \left(1-\frac{x y}{v_{2}}\right)^{n} H_{x}=\left(1-\frac{x y}{v_{1}}\right)^{n-1}\left(1-\frac{x y}{v_{1} v_{2}}\right)=0 .
\end{aligned}
$$

The common zeroes of this system (for $n>1$ ) lie exactly on the hyperbola $Y_{0}^{1}=\{y=$ $\left.v_{1} / x\right\}$. Notice that the partial derivative $H_{x}$ vanishes, in addition, on the hyperbola $y=$ $v_{1} v_{2} / x$. Hence, the points of this hyperbola are zeroes of the derivative $y^{\prime}$ of the solutions of (5.1) passing through these points. Of course, this can be checked by direct substitution.

Remark 1. The fact that $H$ is the first integral of the equation (5.1), and hence its level curves must be locally graphs of a regular function at each finite point, does not exclude by itself possible critical points of $H$-compare the points of the hyperbola $Y_{0}^{1}$. 
Remark 2. Instead of the rational equation (7.3) we can consider the equivalent polynomial equation

$$
W(x, y)=y\left(1-\frac{x y}{v_{1}}\right)^{n}-y_{0}\left(1-\frac{x y}{v_{2}}\right)^{n+1}=0
$$

The advantage of (7.3) is that the initial value $y_{0}$ appears there just as the right hand side.

Now, differentiating (7.4) with respect to $x$ and $y$ and equating to zero we get the following system of equations:

$$
\begin{aligned}
& W_{x}=\left(1-\frac{x y}{v_{1}}\right)^{n}+\frac{n x y}{v_{1}}\left(1-\frac{x y}{v_{1}}\right)^{n-1}-\frac{(n+1) x y_{0}}{v_{2}}\left(1-\frac{x y}{v_{2}}\right)^{n}=0, \\
& W_{y}=\frac{n y^{2}}{v_{1}}\left(1-\frac{x y}{v_{1}}\right)^{n-1}-\frac{(n+1) y y_{0}}{v_{2}}\left(1-\frac{x y}{v_{2}}\right)^{n}=0 .
\end{aligned}
$$

Multiplying the first equation by $y$ and the second by $x$ and subtracting, we get

$$
y\left(1-\frac{x y}{v_{1}}\right)^{n}=0
$$

So either $y=0$ or the point $(x, y)$ belongs to $Y_{0}^{1}$. If $y=0$ then the second equation above is satisfied, while the first equation gives $x=v_{2} /\left((n+1) y_{0}\right)$. So the function $W(x, y)$ has an additional critical point, not on the hyperbola $Y_{0}^{1}$. Notice, however, that for any $y_{0} \neq 0$ this point does not belong to the solution curve of (7.4), while for $y_{0}=0$ it is at infinity.

\subsection{Singularities of solutions of $\frac{d y}{d x}=c x y^{3}+y^{2}$}

The only "fixed" singularity of equation (5.1) is the origin $x=0$. Let us start with the "movable" singularities $x_{0} \neq 0$ of solutions (compare with the general results of Section 2).

Proposition 7.1. For $y_{0} \neq 0$ the solution $y\left(y_{0}, x\right)$ has the only movable singularity at the point $x_{0}\left(y_{0}\right)=\kappa / y_{0}$, where

$$
\kappa=-v_{2}\left(\frac{v_{2}}{v_{1}}\right)^{n}=-\frac{2}{1-\delta}\left(\frac{1-\delta}{1+\delta}\right)^{n} \approx-2 e
$$

for $\delta$ small. Exactly one local branch of $y\left(y_{0}, x\right)$ takes an infinite value and has a ramification of order 2 at $x_{0}\left(y_{0}\right)$, while the other $n-1$ local branches are regular at this point and take there $n-1$ different finite values. 
Proof. Denoting, as above, $1 / y$ by $u$ we get from (7.3)

$$
y_{0}=\frac{\left(u-x / v_{1}\right)^{n}}{\left(u-x / v_{2}\right)^{n+1}} \text {. }
$$

Substituting here $u=0$ (and assuming $x \neq 0$ and so cancellation is possible) we get $x_{0}\left(y_{0}\right)=\kappa / y_{0}$. So the local branch of $y\left(y_{0}, x\right)$ can take an infinite value only at the point $x_{0}\left(y_{0}\right)=\kappa / y_{0}$.

To find the order of branching of $u$ as a function of $x$, we write the series expansion of (7.5) as follows:

$$
x=\frac{\kappa}{y_{0}} \frac{\left(1-u v_{1} / x\right)^{n}}{\left(1-u v_{2} / x\right)^{n+1}}=\frac{\kappa}{y_{0}}\left(1+\frac{u}{x}\left[(n+1) v_{2}-n v_{1}\right]+A\left(\frac{u}{x}\right)^{2}+\cdots\right) .
$$

Since $(n+1) v_{2}-n v_{1}=0$, we can rewrite the last expression as

$$
x=\frac{\kappa}{y_{0}}\left(1+A\left(\frac{u}{x}\right)^{2}+\cdots\right), \quad x-\frac{\kappa}{y_{0}}=B\left(\frac{u}{x}\right)^{2}(1+\cdots) .
$$

Although (7.7) contains $x$ on both sides, it shows that $x-\kappa / y_{0}$ as a function of $u$ has a second order zero at $u=0$, and hence $u$ as a function of $x$ has at $x_{0}=\kappa / y_{0}$ a second order branching. (We do not prove that the coefficient $B$ above is different from zero, since this fact was stated in general form in Section 2.) This completes the description of the branch passing through the point $\left(x_{0}\left(y_{0}\right), \infty\right)$.

Any other branch of the solution $y\left(y_{0}, x\right)$ (i.e. of the curve $\left.H(x, y)=y_{0}\right)$ over $x_{0}$ is regular, since by Lemma 7.1 all the singularities of $H$ belong to the level curve $H=0$. Since for any fixed $x$ the total number of solutions of $H(x, y)=y_{0}$ with respect to $y$, counted with multiplicities, is $n+1$, and since the multiplicity of the singular branch is 2 , there are exactly $n-1$ regular local branches of the curve $H(x, y)=y_{0}$ over $x_{0}$. This completes the proof of the proposition.

Remark. Exactly as in Sections 3 and 4, we can use the series (7.7) to analyze the local structure of the singularities of the Poincaré mapping. Indeed, for a fixed $x=x_{0}$ we can rewrite (7.7) as

$$
y_{0}-\frac{\kappa}{x_{0}}=D\left(\frac{u}{x_{0}}\right)^{2}(1+\cdots),
$$

and we get a second order zero of $y_{0}-\kappa / x_{0}$ as a function of $u\left(x_{0}\right)$ and a second order ramification of $u\left(x_{0}\right)$ as a function of $y_{0}$.

The next step is to investigate the structure of the fixed singularity $x=0$.

Proposition 7.2. For $y_{0} \neq 0$ the solution $y\left(y_{0}, x\right)$ has over $x=0$ two local components: the regular one, passing through the point $\left(0, y_{0}\right)$, and the singular one, passing through the point $(0, \infty)$. The singular component is represented by the Puiseux series

$$
\frac{1}{y(x)}=u(x)=\frac{1}{v_{1}} x+\mu x^{1+1 / n}+\cdots,
$$


with

$$
\mu=y_{0}^{1 / n}\left(\frac{1}{2 n+1}\right)^{1+1 / n} \approx \frac{1}{2 n} y_{0}^{\frac{1}{n}} .
$$

In particular, the local monodromy acts as a cyclic permutation of the infinite branches.

Proof. Let us rewrite equation (7.3) in the form

$$
y_{0}\left(u-\frac{x}{v_{2}}\right)^{n+1}=\left(u-\frac{x}{v_{1}}\right)^{n} .
$$

We have to find the Puiseux expansion of the curve given by (7.10) at the point $(u, x)=$ $(0,0)$. We use the following "Ansatz":

$$
u(x)=\frac{1}{v_{1}} x+\mu x^{v}+\cdots .
$$

Substituting (7.11) into (7.10) we get

$$
y_{0}\left[\left(\frac{1}{v_{1}}-\frac{1}{v_{2}}\right) x+\mu x^{v}+\cdots\right]^{n+1}=\mu^{n} x^{\nu n}+\cdots .
$$

Comparing the leading degrees and coefficients, we obtain

$$
y_{0}\left(\frac{1}{v_{1}}-\frac{1}{v_{2}}\right)^{n+1} x^{n+1}+\cdots=\mu^{n} x^{v n}+\cdots,
$$

and hence

$$
\mu=y_{0}^{1 / n}\left(\frac{1}{v_{1}}-\frac{1}{v_{2}}\right)^{(n+1) / n}=y_{0}^{1 / n}(-\delta)^{1+1 / n} \approx \frac{1}{2 n} y_{0}^{1 / n}, \quad v=1+\frac{1}{n} .
$$

Let us illustrate the above computations by writing explicitly the solution $y$ in the case $n=1$, i.e. for $c=c_{1}=2 / 9$. Equation (7.4) takes the form

$$
W(x, y)=y\left(1-\frac{x y}{v_{1}}\right)-y_{0}\left(1-\frac{x y}{v_{2}}\right)^{2}=0 .
$$

Taking into account that for $n=1$ we have $v_{1}=-3, v_{2}=-3 / 2$, we get the following quadratic equation for $y$ :

$$
\frac{1}{3} x\left(\frac{4}{3} x y_{0}-1\right) y^{2}+\left(\frac{4}{3} x y_{0}-1\right) y+y_{0}=0 .
$$

Solving it we get

$$
y=-\frac{3}{2 x}\left(-1 \pm \frac{1}{\sqrt{1-\frac{4}{3} x y_{0}}}\right) .
$$

This function has a double ramified pole at $x=3 /\left(4 y_{0}\right)$, a branch with a usual pole at $x=0$, and a regular branch passing through $\left(0, y_{0}\right)$. 


\subsection{Global ramification of solutions}

According to Propositions 7.1 and 7.2, there are only two singularities of the solution $y\left(y_{0}, x\right)$ for $y_{0} \neq 0$ : the fixed singularity at $x=0$ and the movable singularity of one of the branches of $y$ at $x_{0}\left(y_{0}\right)=\kappa / y_{0}$. The original local branch at $x=0$ of $y\left(y_{0}, x\right)$ is regular at the origin. Hence, it can be analytically extended as a regular single-valued function into the disk $\mathcal{D}=D_{\left|\kappa / y_{0}\right|}$, centered at $x=0$.

Lemma 7.2. The regular branch of $y\left(y_{0}, x\right)$ on the disk $\mathcal{D}$ has a singularity at the boundary point $\kappa / y_{0}$.

Proof. Take $y_{0}$ positive. By our choice of the parameter $c \approx 1 / 4$ we have $c>0$. Therefore, the right hand side of (5.1) is positive, and bounded from below by $y^{2}$, and hence its solution blows up in finite time on the semi-axis $x>0$. By Proposition 7.1, this happens exactly at the point $x_{0}\left(y_{0}\right)=\kappa / y_{0}$. This proves the lemma for $y_{0}$ positive. Now, as $y_{0}$ moves along the circle $\left|y_{0}\right|=$ const, the singularity $x_{0}\left(y_{0}\right)=\kappa / y_{0}$ of the regular single-valued function $y\left(y_{0}, x\right)$ on the disk $\mathcal{D}$ moves along the boundary of this disk. Since $y\left(y_{0}, x\right)$ analytically depends on $y_{0}$, the point $x_{0}\left(y_{0}\right)$ remains its singularity. This completes the proof.

Let the value $y_{0} \neq 0$ be fixed. Consider the loop $\omega$ in the $x$-plane following the straight line segment from 0 to the singular point $x_{0}\left(y_{0}\right)$, then going around this point in a counter-clockwise direction along a small circle, and then returning to 0 along the same straight line segment.

Lemma 7.3. The regular branch at $x=0$ of the solution $y\left(y_{0}, x\right)$ analytically continued along the loop $\omega$ returns at $x=0$ to one of the infinite branches of the solution.

Proof. Since $y\left(y_{0}, x\right)$ has a second order ramification at $x_{0}$, after one turn around this point we get another branch of the solution. When we return to zero, we stay on this new branch, different from the initial (regular) one. But by Proposition 7.2 all the branches, except the initial one, tend to $\infty$ at $x=0$.

Now we have enough tools to prove one of the main properties of the solutions of (5.1), as given by the first integral $H(x, y)=y_{0}$ :

Theorem 7.1. For each $y_{0} \neq 0$ the solution curve $Y_{y_{0}}=\left\{H(x, y)=y_{0}\right\}$ is irreducible. Analytic continuation of the local solution $y\left(y_{0}, x\right)$ at zero along the loop $\omega$ and then several turns around zero transform this local branch to each of the remaining $n$ branches of $Y_{y_{0}}$.

Proof. By Lemma 7.3 continuation along $\omega$ transforms the local regular branch of $y\left(y_{0}, x\right)$ at zero into one of the infinite branches. By Proposition 7.2, each turn around zero results in a cyclic permutation of the $n$ infinite branches. Hence, in at most $n$ turns any other infinite branch can be obtained. 


\section{Poincaré mapping, $c=c_{n}$}

The first integral for the Abel equation (5.1) with $c=c_{n}$, as computed in Section 7, equation (7.3), has the form

$$
y_{0}=\frac{y\left(1-x y / v_{1}\right)^{n}}{\left(1-x y / v_{2}\right)^{n+1}} .
$$

Substituting here $x=b$ we get an algebraic equation

$$
y_{0}=\frac{y\left(1-b y / v_{1}\right)^{n}}{\left(1-b y / v_{2}\right)^{n+1}}
$$

which expresses the relation between $y$ and $y_{0}$ in the Poincaré mapping from $a$ to $b$. In particular, read as an expression for $y_{0}$ through $y$, (8.2) represents $y_{0}$ as a rational function of $y$. However, such a straightforward interpretation of (8.2) is somewhat misleading, since for each $y_{1}$ the continuation path from $b$ to 0 must be specified, avoiding singularities of the solution $y\left(b, y_{1}\right)$.

Indeed, without taking this into account, (8.2) produces some peculiar phenomena. In particular, the derivative $d y_{0} / d y$ at $y=v_{1} / b$ is zero by (8.2). On the other hand, the derivative of the Poincaré mapping cannot vanish (since the Poincaré mapping is always locally invertible).

The correct interpretation of (8.2) is that the algebraic solution curve given by (8.1) contains the points $\left(0, y_{0}\right)$ and $(b, y)$. However, an analytic continuation of this solution from $(b, y)$ along a given path can be either $y_{0}$ or $\infty$, according to the results of Section 7 . In particular, the point $\left(b, v_{1} / b\right)$ belongs to the hyperbola $y=v_{1} / x$, and its analytic continuation to $x=0$ is $\infty$ for any path. Therefore, we cannot come from $\left(b, v_{1} / b\right)$ to $(0,0)$, whichever continuation path is taken, and the vanishing derivative above is not the derivative of any "true" Poincaré mapping.

\subsection{Example: $n=1$}

In this paper we provide a complete description of the ramification structure of the Poincaré mapping only in one simple example: for $n=1$. We also assume $b=1$. In this case equations (8.1) and (8.2) take the form

$$
\begin{aligned}
& y_{0}=\frac{y(1+x y / 3)}{(1+2 x y / 3)^{2}}, \\
& y_{0}=\frac{y(1+y / 3)}{(1+2 y / 3)^{2}} .
\end{aligned}
$$

Solving the first equation with respect to $y$ we get

$$
y=-\frac{3}{2 x}\left(-1 \pm \frac{1}{\sqrt{\frac{4}{3} x y_{0}-1}}\right),
$$


and substituting $x=1$ we obtain the Poincaré mapping from 0 to 1 as

$$
y=-\frac{3}{2}\left(-1 \pm \frac{1}{\sqrt{1-\frac{4}{3} y_{0}}}\right) .
$$

Now, the singular point of the solution is at $x=3 /\left(4 y_{0}\right)$. The singular point $y_{0}=3 / 4$ of the Poincaré mapping corresponds (in agreement with the description of Section 3 above) to the singularity of the solution being at $x=1$.

Following Section 3, let us consider the values of $y_{0}$ for which the singular point of the solution $x=3 /\left(4 y_{0}\right)$ crosses the continuation segment [0, 1]. They form the segment $J=\left[\frac{3}{4}, \infty\right]$. Consequently, for $y_{0} \in \mathbb{C} \backslash J$ the Poincaré mapping, as given by the branch of (8.5) with the value 0 for $y_{0}=0$, corresponds to the continuation segment $[0,1]$. As $y_{0}$ crosses $J$, the continuation path must be deformed as described in Section 3. After two turns we return to the same branch-this is specific to the case $n=1$, where the infinite branch of the solution over 0 does not ramify.

\section{9. "Limit cycles", $c=c_{n}$}

Theorem 9.1. For $c=c_{n}=\frac{1}{4}\left(1-\frac{1}{(2 n+1)^{2}}\right)$ and $b \neq 0$ equation (5.1) has $n$ different "limit cycles", i.e. local solutions $y^{j}(x)$ at the origin, $j=1, \ldots, n$, and paths $s^{j}$ from 0 to $b$, such that each $y^{j}(x)$ when analytically continued along $s^{j}$ satisfies $y(0)=y(b)$. The ramification structure of these "limit cycles", in particular, the paths $s^{j}$, approximates that of the case $c=1 / 4$ as $n$ tends to infinity.

Proof. Let us rewrite equation (5.6) for the "limit cycles" in the form

$$
\left(1-\frac{b y_{0}}{v_{1}}\right)^{n}-\left(1-\frac{b y_{0}}{v_{2}}\right)^{n+1}=0 .
$$

First, we have to analyze the algebraic equation (9.1). Notice that (9.1) depends only on the product $b y_{0}$. Denoting $b y_{0}$ by $\kappa$ we get

$$
\left(1-\frac{\kappa}{v_{1}}\right)^{n}-\left(1-\frac{\kappa}{v_{2}}\right)^{n+1}=0 .
$$

Denoting the roots of (9.2) by $\kappa_{0}, \ldots, \kappa_{n}$ we get for any $b \neq 0$ the corresponding solutions $y_{0}^{j}=b / \kappa_{j}, j=0, \ldots, n$, of $(9.1)$.

Lemma 9.1. For large enough integer values of $n$ equation (9.2) has $n+1$ distinct complex solutions $\kappa_{0}, \ldots, \kappa_{n}$.

Proof. Indeed, equating to zero the derivative of (9.2) we get

$$
-\frac{n}{v_{1}}\left(1-\frac{\kappa}{v_{1}}\right)^{n-1}+\frac{n+1}{v_{2}}\left(1-\frac{\kappa}{v_{2}}\right)^{n}=0 .
$$


Multiplying this equation by $\left(v_{1} / n\right)\left(1-\kappa / v_{1}\right)$ and adding it to (9.2) we obtain, after some transformations, the equation

$$
\left(1-\frac{\kappa}{v_{2}}\right)^{n}\left[-2-\frac{1}{n}-\frac{(n+1)^{2} \kappa}{2 n+1}\right]=0 .
$$

It has one root $\tilde{\kappa}=v_{2}=-2+\frac{1}{n+1}$ of multiplicity $n$, and another simple root $\hat{\kappa}=$ $(2 n+1)^{2} /\left(n(n+1)^{2}\right)$. The first root clearly is not a root of (9.2). Substituting into (9.2) the second root of (9.3) we get

$$
\left(1-\frac{(2 n+1)^{2}}{n(n+1)^{2}}\right)^{n}-\left(1-\frac{(2 n+1)^{3}}{n(n+1)^{3}}\right)^{n+1}
$$

which for $n$ large enough gives $(1-4 / n+\cdots)^{n}-(1-8 / n+\cdots)^{n+1} \approx e^{-4}-e^{-8} \neq 0$. Therefore, (9.2) and its derivative do not have common roots. Hence, all the roots of (9.2) are simple, different from one another, and their number is $n+1$. This completes the proof of the lemma.

Consider now the local solutions $y^{j}(x)=y\left(y_{0}^{j}, x\right)$ at the origin satisfying $y^{j}(0)=$ $y_{0}^{j}, j=0, \ldots, n$, where $y_{0}^{j}, j=0, \ldots, n$, are all the distinct solutions of (9.1) given by Lemma 9.1. Analytic continuation of $y^{j}(x)$ gives an algebraic curve $Y_{y_{0}^{j}}$ satisfying equation (5.4) with $y_{0}=y_{0}^{j}$. Now equation (9.1) says exactly that the points $\left(0, y_{0}^{j}\right)$ and $\left(b, y_{0}^{j}\right)$ belong to $Y_{y_{0}^{j}}$. By Theorem 7.1 this curve is irreducible, and we can pass from the point $\left(0, y_{0}^{j}\right)$ to the point $\left(b, y_{0}^{j}\right)$ via analytic continuation of the local branch $y^{j}(x)=y\left(y_{0}^{j}, x\right)$ at the origin along a certain path $s^{j}$, as described in Theorem 9.1.

As $n$ tends to infinity, $c_{n}$ tends to $1 / 4$. By the analytic dependence on the parameter, the solutions and the Poincaré mapping of the Abel equation with $c=c_{n}$ approximate that of the limit one on each compact domain. Hence the same is also true for the ramification structure of the "limit cycles" over the paths $s^{j}$ of "bounded complexity". This completes the proof of Theorem 9.1.

Acknowledgments. The first author expresses his gratitude to the Weizmann Institute for providing a financial support for a visit during which this article was initiated. The third author expresses his gratitude to the University Paris 6 and to the IHES for providing a financial support for a visit during which this article was continued. The work of the second and third authors has also been supported by the ISF Grants No. 264/02 and 979/05, by the BSF Grant No. 2002243, and by the Minerva Foundation.

\section{References}

[1] Abel, N.: Oeuvres complètes. S. Lie and L. Sylow (eds.), Grondahl, Christiania (1881). JFM 13.0020.01 MR 0963862

[2] Alwash, M. A. M.: On a condition for a centre of cubic non-autonomous equations. Proc. Roy. Soc. Edinburgh Sect. A 113, 289-291 (1989) Zbl 0685.34025 MR 1037734 
[3] Alwash, M. A. M.: Word problems and the centers of Abel differential equations. Ann. Differential Equations 11, 392-396 (1995) Zbl 0843.34034 MR 1384574

[4] Alwash, M. A. M.: On the composition conjectures. Electronic J. Differential Equations 2003, no. 69, 4 pp. Zbl 1054.34047 MR 1993777

[5] Alwash, M. A. M., Lloyd, N. G.: Non-autonomous equations related to polynomial two-dimensional systems. Proc. Roy. Soc. Edinburgh Sect. A 105A, 129-152 (1987) Zbl 0618.34026 MR 0890049

[6] Bautin, N. N.: On the number of limit cycles which appear with the variation of coefficients from an equilibrium position of focus or center type. Mat. Sb. 30, 181-196 (1952) (in Russian); English transl.: Amer. Math. Soc. Transl. 5, 396-413 (1962) Zbl 0059.08201 MR 0045893

[7] Blinov, M., Briskin, M., Yomdin, Y.: Local center conditions for a polynomial Abel equation. J. Anal. Math., to appear

[8] Briskin, M., Francoise, J.-P., Yomdin, Y.: The Bautin ideal of the Abel equation. Nonlinearity 11, 431-443 (1998) Zbl 0905.34051 MR 1617943

[9] Briskin, M., Francoise, J.-P., Yomdin, Y.: Center conditions, compositions of polynomials and moments on algebraic curves. Ergodic Theory Dynam. Systems 19, 1201-1220 (1999) Zbl 0990.34017 MR 1721616

[10] Briskin, M., Francoise, J.-P., Yomdin, Y.: Center condition II: Parametric and model center problems. Israel J. Math. 118, 61-82 (2000) Zbl 0989.34021 MR 1776076

[11] Briskin, M., Francoise, J.-P., Yomdin, Y.: Center condition III: Parametric and model center problems. Israel J. Math. 118, 83-108 (2000) Zbl 0989.34022 MR 1776077

[12] Briskin, M., Francoise, J.-P., Yomdin, Y.: Generalized moments, center-focus conditions and compositions of polynomials. In: Operator Theory, System Theory and Related Topics, Oper. Theory Adv. Appl. 123, Birkhäuser, 161-185 (2001) Zbl 1075.34509 MR 1821911

[13] Briskin, M., Roytvarf, N., Yomdin, Y.: Center conditions at infinity. Submitted

[14] Cheb-Terrab, E. S., Roche, A. D.: Abel ODEs: Equivalence and integrable classes. Comput. Phys. Comm. 130, 204-231 (2000)

[15] Christopher, C.: Abel equations: composition conjectures and the model problem. Bull. London Math. Soc. 32, 332-338 (2000) Zbl 1047.34019 MR 1750484

[16] Corless, R., Gonnet, G., Hare, D., Jeffrey, D., Knuth, D.: On the Lambert W function. Adv. Comput. Math. 5, 329-359 (1996) Zbl 0863.65008 MR 1414285

[17] Devlin, J.: Word problems related to periodic solutions of a nonautonomous system. Math. Proc. Cambridge Philos. Soc. 108, 127-151 (1990) Zbl 0726.34026 MR 1049766

[18] Devlin, J.: Word problems related to derivatives of the displacement map. Math. Proc. Cambridge Philos. Soc. 110, 569-579 (1991) Zbl 0744.34032 MR 1120490

[19] Devlin, J., Lloyd, N. G., Pearson, J. M.: Cubic systems and Abel equations. J. Differential Equations 147, 435-454 (1998) Zbl 0911.34020 MR 1633961

[20] Francoise, J.-P.: Local bifurcations of limit cycles, Abel equations and Liénard systems (notes by Hur Seok). In: Normal Forms, Bifurcations and Finiteness Problems in Differential Equations, Y. Iliashenko et al. (eds.), NATO Sci. Ser. II Math. Phys. Chem. 137, Kluwer, 187-209 (2004) MR 2083247

[21] Francoise, J.-P., Roytvarf, N., Yomdin, Y.: Analytic continuation and fixed points of the Poincaré mapping for polynomial Abel equation. arXiv math.CA/0603534

[22] Francoise, J.-P., Yomdin, Y.: Bernstein inequality and applications to analytic geometry and differential equations. J. Funct. Anal. 146, 185-205 (1997) Zbl 0869.34008 MR 1446379

[23] Francoise, J.-P., Yomdin, Y.: Projection of analytic sets and Bernstein inequalities. In: Singularities Symposium—Łojasiewicz 70, B. Jakubczyk et al. (eds.), Banach Center Publ. 44, Inst. Math., Polish Acad. Sci., Warszawa, 103-108 (1998) Zbl 0915.30002 MR 1677339 
[24] Gasull, H. A., Llibre, J.: Limit cycles for a class of Abel equations. SIAM J. Math. Anal. 21, 1235-1244 (1990) Zbl 0732.34025 MR 1062402

[25] Golubev, V.: Lectures on Analytic Theory of Differential Equations. Gostekhizdat, Moscow (1950) (in Russian) Zbl 0038.24201 MR 0042571

[26] Il'yashenko, Yu.: An example of equations $d w / d z=P_{n}(z, w) / Q_{n}(z, w)$ having a countable number of limit cycles and arbitrarily high Petrovskiı̌-Landis genus. Mat. Sb. 122, 388-404 (1969) (in Russian) Zbl 0214.09404

[27] Il'yashenko, Yu.: Centennial history of Hilbert's 16th problem. Bull. Amer. Math. Soc. (N.S.) 39, 301-354 (2002) Zbl 1004.34017 MR 1898209

[28] Il'yashenko, Yu.: Hilbert-type numbers for Abel equations, growth and zeros of holomorphic functions. Nonlinearity 13, 1337-1342 (2000) Zbl 1016.34028 MR 1767962

[29] Ince, E. L.: Ordinary Differential Equations. Dover (1944) Zbl 0063.02971 MR 0010757

[30] Khovanskiü, A. G.: Fewnomials. Amer. Math. Soc., Providence, RI (1991) Zbl 0728.12002 MR 1108621

[31] Li, C., Li, W., Llibre, J., Zhang, Z.: New families of centers and limit cycles for polynomial differential systems with homogeneous nonlinearities. Ann. Differential Equations 19, 302 317 (2003) Zbl 1055.34060 MR 2018297

[32] Lins Neto, A.: On the number of solutions of the equation $x^{\prime}=P(x, t)$ for which $x(0)=$ $x$ (1). Invent. Math. 59, 67-76 (1980) Zbl 0448.34012 MR 0575082

[33] Liouville, R.: Sur une équation différentielle du premier ordre. Acta Math. 27, 55-78 (1903) JFM 34.0364.03 MR 1554972

[34] Lloyd, N. G.: The number of periodic solutions of the equation $z^{\prime}=z^{N}+p_{1}(t) z^{N-1}+\cdots+$ $p_{n}(t)$. Proc. London Math. Soc. 27, 667-700 (1973) Zbl 0273.34042 MR 0333356

[35] Loray, F.: Sur les théorèmes I et II de Painlevé. In: Geometry and Dynamics, Contemp. Math. 389, Amer. Math. Soc., 165-190 (2005) Zbl 0095.06405 MR 0114953

[36] Murphy, G. M.: Ordinary Differential Equations and Their Solutions. Van Nostrand, Princeton (1960) Zbl 0095.06405 MR 0114953

[37] Painlevé, P.: Oeuvres. R. Gérard, G. Reeb et A. Sec (eds.), Éditions du CNRS, Paris (19731975)

[38] Pakovich, F., Roytvarf, N., Yomdin, Y.: Cauchy-type integrals of algebraic functions and a tangential center-focus problem for Abel equations. Israel J. Math., to appear

[39] Petrovskiur, I., Landis, E.: On the number of limit cycles of the equation $d y / d x=$ $P(x, y) / Q(x, y)$ where $P(x, y)$ and $Q(x, y)$ are polynomials of second degree. Mat. Sb. 37, 209-250 (1955) (in Russian) Zbl 0065.07202 MR 0073004

[40] Risler, J.-J.: Additive complexity and zeros of real polynomials. SIAM J. Comput. 14, 178183 (1985) Zbl 0562.12020 MR 0774937

[41] Yang, L. J., Tang, Y.: Some new results on Abel equations. J. Math. Anal. Appl. 261, 100-112 (2001) Zbl 0995.34031

[42] Yomdin, Y.: Global finiteness properties of analytic families and algebra of their Taylor coefficients. In: The Arnoldfest (Toronto, 1997), Fields Inst. Comm. 24, Amer. Math. Soc., Providence, RI, 527-555 (1999) Zbl 0944.34003 MR 1733591 